\title{
Automatic Exudate Detection Using Eye Fundus Image Analysis Due to Diabetic Retinopathy
}

\author{
Nasr Y. Gharaibeh ${ }^{1}$, Ma'moun A. Al-Smadi ${ }^{1} \&$ Mohammad Al-Jarrah ${ }^{2}$ \\ ${ }^{1}$ Electrical Eng. Dep., AlBalqa Applied Univ., Salt, Jordan \\ ${ }^{2}$ Computer Eng. Dep., Yarmouk University, Irbid, Jordan \\ Correspondence: Mohammad Al-Jarrah, Computer Eng. Dep., Yarmouk University, Irbid, Jordan. E-mail: \\ jarraj@yu.edu.jo
}

Received: January 28, $2014 \quad$ Accepted: March 13, $2014 \quad$ Online Published: March 20, 2014

doi:10.5539/cis.v7n2p48 URL: http://dx.doi.org/10.5539/cis.v7n2p48

\begin{abstract}
Diabetic retinopathy (damage to the retina) is a disease caused by complications of diabetes, which can eventually lead to blindness. It is an ocular manifestation of diabetes, a systemic disease, which affects up to 80 percent of all patients who have had diabetes for 10 years or more. Despite these intimidating statistics, research indicates that at least $90 \%$ of these new cases could be reduced if there was proper and vigilant treatment and monitoring of the patient eyes. The longer a person has diabetes, the higher his or her chances of developing diabetic retinopathy. In this paper, we introduced a new method for eye fundus image analysis, based on exudate segmentation. The proposed algorithm detects the existence of exudates and measures its distribution. In this paper, we classified images of eye fundus into no-exudate or have exudates. This initial classification helps physicians to initiate a treatment process for infected patients. The algorithm is tested using DIARETDB0. The results proved the reliability and robustness of algorithm.
\end{abstract}

Keywords: exudates, eye fundus, diabetic disease, image segmentation, diabetic retinopathy

\section{Introduction}

Medical image analysis is an area of research that is currently attracting many researchers in all aspect of health and medications (Schneider, Rasband, \& Eliceiri, 2012; Walter, Klein, Massin, \& Erginay, 2002; Tasman \& Jaeger, 2001). This field involves the study of digital images in order to provide computational tools, which will assist the quantification and visualization of interesting pathology and anatomical structures. The progress achieved in this field over recent years has significantly improved the type of medical care that is available to patients. Physicians have advanced diagnostic tools to evaluate their patients' cases in order to plan different forms of medical management and monitor the progress more efficiently (Schneider et al., 2012). In particular, the investigation and the analysis of eye fundus image in the object of determining the impact of diabetes on the vision system is becoming more significant (Walter et al., 2002; Cunha-Vaz, 1998).

Diabetes is a rapidly increasing worldwide problem, which is characterized by defective metabolism of glucose that causes long-term dysfunction and failure of various organs. The most common complications of diabetes are diabetic retinopathy (DR), and diabetic macular edema (DME), which are considered one of the primary causes of blindness and visual impairment in adults (Cunha-Vaz, 1998; Tasman \& Jaeger, 2001). The rapid increase of diabetes pushes the limits of the current DR/DME screening capabilities for which the digital imaging of the eye fundus and automatic or semi-automatic image analysis algorithms provide a potential solution. The retina lesions and abnormalities that can be detected using the methods of eye fundus images are hard exudate, soft exudates, microaneurysms, and hemorrhages (Walter et al., 2002; Cunha-Vaz, 1998; Geetan, Acharya, \& Ng, 2008).

Exudates appeared as bright yellow-white deposits on the retina due to the leakage of lipid from abnormal vessels (Tasman \& Jaeger, 2001). Their shape and size varies with the different retinopathy stages. These lesions are associated with numerous retinal vascular diseases, including diabetic macular edema DME, diabetic retinopathy DR, hypertensive retinopathy, retinal venous obstruction, retinal arterial micoaneurysms, radiation retinopathy, Coat's disease, and capillary hemangioma of the retina (i.e. von Hippel's lesion). Exudation is a risky case because it can lead to severe visual loss when occurring in the central macular region. Thus, such 
lesions must be detected and proper medical intervention must be taken to avoid damages to the patient visual acuity (Welfera, Scharcanskia, \& Marinho, 2010). Many researchers tackled exudate detection and classification. The following paragraphs summarize the recent papers in this field.

Niemeijer, van Ginneken, Russell, and Abramoff (2007) proposed a pixel classification scheme based on k-nearest neighbour classification to detect and differentiate hard, soft exudates, and drusen. The high probability regions were pruned to find the true bright lesions by extracting descriptive features for each region and applying the KNN classification. Finally, a linear classifier was used to classify the detected true bright lesions to hard, soft exudates, or drusen. A feature-based classification scheme was also proposed by Xu and Luo (2009), where a feature combination based on stationary wavelet transform and gray level co-occurrence matrix was used to characterize the textual properties of hard exudates. The pixel level classification was conducted using a support vector machine.

Agurto et al. (2010) proposed a method, where the lesion map is not employed directly, but rather inferred by a set of frequency domain based features that describe the image as a whole. However, the described technique is only for the diagnosis of DR. Sanchez, Garcia, Mayo, Lopez, and Hornero (2009) presented a more sophisticated technique based on image normalization and distribution analysis. Walter, Klein, Massin, and Erginay (2002) proposed a method employing greyscale morphological operators to identify all structures with predictable shapes (such as vessels). These structures are removed from the image so that exudates can be identified.

Garcia, Sanchez, Lopez, Abasolo, and Hornero (2009) presented a classification method which build a feature vector for each pixel or pixel cluster, which are then classified by employing a neural network approach. The suggested neural network classifies regions into exudates or not exudates.

In this paper, we proposed an algorithm that detects the existence of exudates and measure its distribution in eye fundus. The algorithm applies two different approaches to detect exudates. The first approach employs color properties for exudates and the second approach utilizes boundary identification to allocate exudates in eye fundus image. The final decision for identifying the exudates is accomplished by intersecting both approaches results. Only exudates identified by both approaches are marked as exudates. This approach reduces miss classification of exudates.

The remaining of this paper is organized as follows. In section 2 we introduced exudate detection algorithm. In section 3, we discussed the implementation of the proposed algorithm and the results. Finally, we concluded the paper in section 4 .

\section{Exudate Detection}

Diabetic retinopathy disease develops exudates in eye fundus. The physicians consider the exudate as one of major indicator of the severity of the diabetic retinopathy (Schneider et al., 2012). In this paper, we employed the advances of image processing science in helping physicians to diagnose diabetic retinopathy based on detecting exudates in color fundus image of the humane retina.

Exudates are a yellow spot usually located in the posterior pole of the fundus (Walter et al., 2002). Eye exudates are made of fluid leaked from blood vessels. Diabetes disease causes the fluid leakage from blood vessels. Uncontrolled diabetes for long time may develop exudates in eye fundus. The exudates start to develop in small size and number. If the diabetes is not controlled or monitored for long time, the size and the number of exudates will increase. The development of exudates in eye fundus may cause blindness. Therefore, detecting and monitoring exudates in eye fundus is important for diabetic patients.

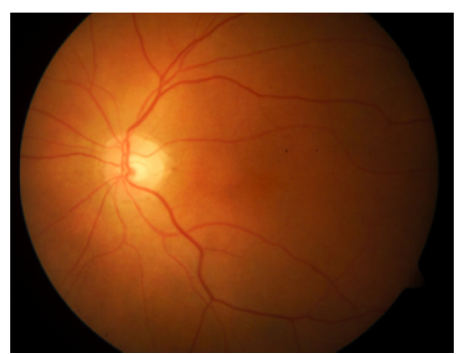

(a)

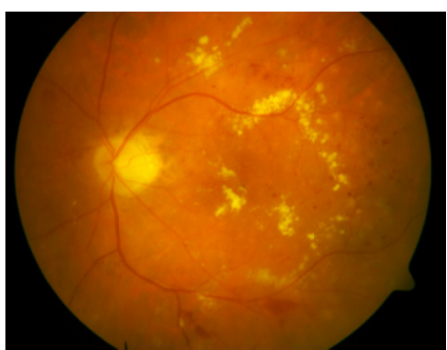

(b)

Figure 1. Color image for eye fundus, (a) eye fundus image without exudate, (b) eye image with exudates 


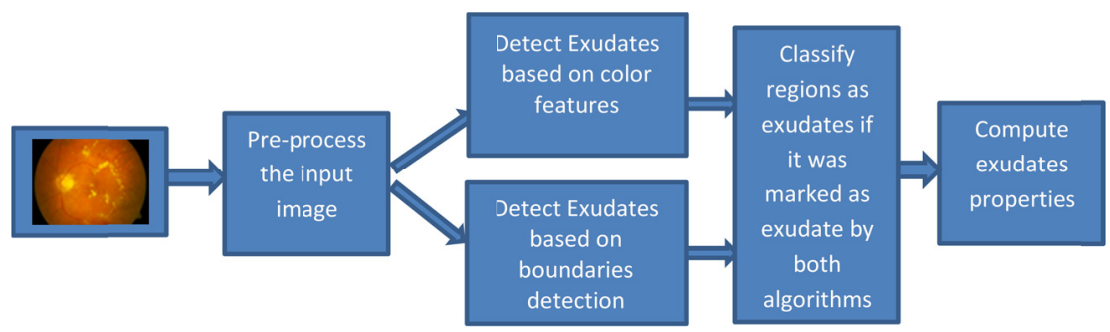

Figure 2. The proposed algorithm to detect exudates in eye image fundus using color features and boundary detection

This paper proposed an algorithm for detecting and evaluating the volume of exudates in eye fundus. The proposed algorithm process a color image for eye fundus. A sample of fundus images is shown Figure 1. The image depicted in Figure 1(b) shows an eye fundus image with clear exudates. The proposed algorithm is divided into three phases. In the first phase, the eye fundus image is normalized. The second phase includes the detection and removal of the optical disk. In the third phase, we proposed new approach to detect exudates based on two different algorithms. Each exudate algorithm marks the detected exudate as candidate exudates. In the first approach we utilized the exudate color features. In the second one, we applied edge and boundary detection. If both algorithms classify a region in the image as a candidate exudate, then this region is classified as exudate. Figure 2 depicts the proposed algorithm to detect exudates in eye image fundus.

\subsection{Image Normalization}

Fundus eye image is acquired using special fundus camera. The fundus eye image is acquired by expert guiding the patient to look at a point visible in back of the camera lens. Even though, the image is acquired in well calibrated and standard environment, the qualities of images vary from on to another. To make sure that the applied algorithm achieves the expected results, the image is preprocessed initially to improve the quality and to standardize the properties of the image. First, the image is mapped to standard size. Then the image filtered to reduce different type of noises mainly salt and pepper. Third image intensity is normalized using histogram equalization. Image intensity normalization is repeated in many stages to reduce the effect of intensity on the algorithm results. After that the image is sharpened to emphasize on the edges. The important part of the preprocessing stage is the extraction of retina fundus from image background. Normally, the captured retinal fundus surrounded with dark background. Figure 1(a) shows the circular wise fundus image in dark background. This step is achieved using edge detection filter followed by contour detection algorithm. Figure 3(b) shows the image after applying the edge detector filter. The contour is developed by start searching from predefined locations. Then the contour is completed to be similar to circle arc. Figure 3(a) shows the results of edge detector after preprocessing steps for eye fundus image. The developed contours that segments the retina fundus form the dark background is shown in Figure 3(c).

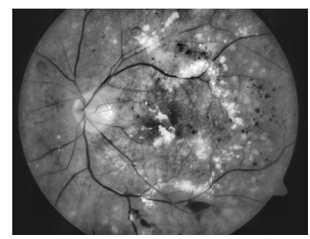

(a)

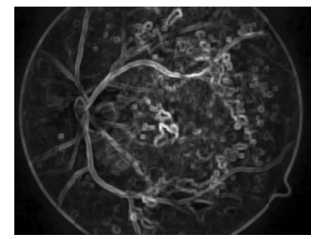

(b)

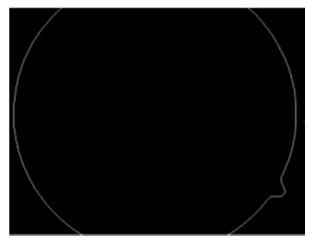

(c)

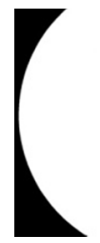

(d)

Figure 3. Extorting eye fundus region (a) Eye fundus image after the preprocessing stage, (b) applying edge detection filter, c) applying counter development to extract retina fundus, and (d) retina fundus area 


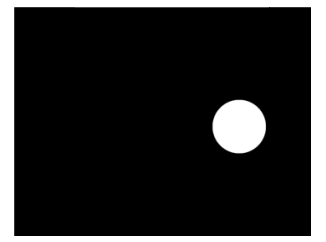

(a)

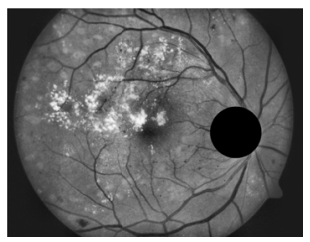

(b)

Figure 4. Optical disc removal from fundus image (a) Optical disc mask, (b) fundus image after removing the optic disk

\subsection{Optical Disc Removal}

Optic disc is small blind area located on the surface of the retina where the fibers of the retina leave the eye and become part of the optic nerve. It is also the entry point for the major blood vessels that supply the retina. Figure 3(a) shows the optic disc as bright elliptic area on the right. The color of the optical disc is very close to exudate color. Thus, the removal of optic disc from the image will help in extracting exudates accurately.

In this paper, we applied simple and fast approach to remove optic disc. First we applied a median filter with a size equal to the average size of optic disc. The optic disc size varies from patient to patient. The optic disc diameter varies from $1.76 \mathrm{~mm}$ to $1.92 \mathrm{~mm}$. Then the center of the optic disc is identified as maximum intensity pixel location in the image. After that, we built an optic disc removal mask through developing a circle with diameter equal to the average size of the optic disc. Figure 4(a) illustrates optic disc removal mask, and Figure 4 (b) depicts the fundus image after removing the optical disc.

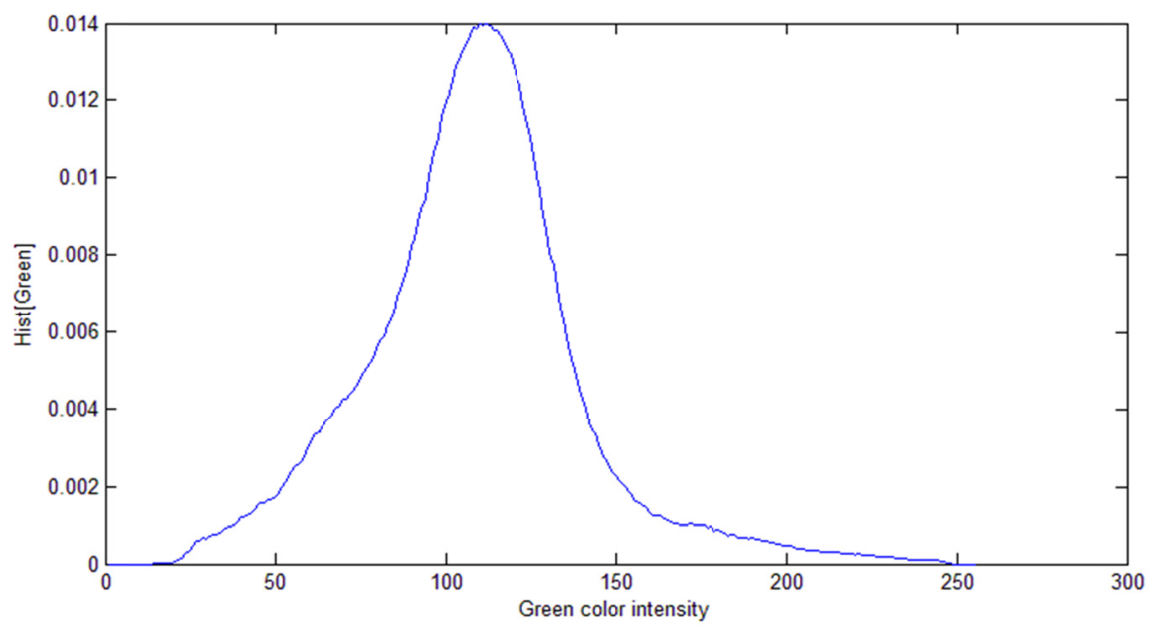

Figure 5. Histogram for green color of the preprocessed eye fundus retina image shown in Figure 3(a)

\subsection{Exudates Detection}

Exudates play a key role in diagnosis of retina diseases. Therefore, detecting exudates with high accuracy is important. In our proposed approach, we used two different techniques to detect exudates. Only exudate that has been marked with both techniques is considered exudate. The first technique relies on color feature of exudates. Exudates are bright yellowish spots surrounded with a darker color. The preprocessed eye fundus image example is shown in Figure 4(b). Figure 5 shows the histogram for green component of the image shown in Figure 1(a). The color mode for image is assumed bimodal where the number of pixels belongs to the background is much higher than the pixels that belong to exudates. Benefit from this feature is that the mean of the whole image is closer to the mean of the pixel that belongs to background. Then, subtracting, the mean of the whole image from the image will produce an image mainly contain exudates with mean shifted with some noise. The next step is removing the noise using median filter. After that, we applied thresholding technique to separate exudate pixels from others. In our case we used upper and lower thresholding. The upper and lower thresholds are adapted to minimize the standard deviation of exudates colors (Liang \& Yin, 2013). In the second technique, we used kirsch filter for edge detection. We applied kirsch filter that utilizes eight compass filters. All eight compass filters are 
applied and the maximum one is considered for final image. Then we utilized contour detection to identify the boundaries of exudates (Arbel'aez \& Fowlkes, 2011). Finally, the regions which are marked by both techniques as exudates are considered exudate. Figure 6 illustrates the proposed algorithm for exudate detection.

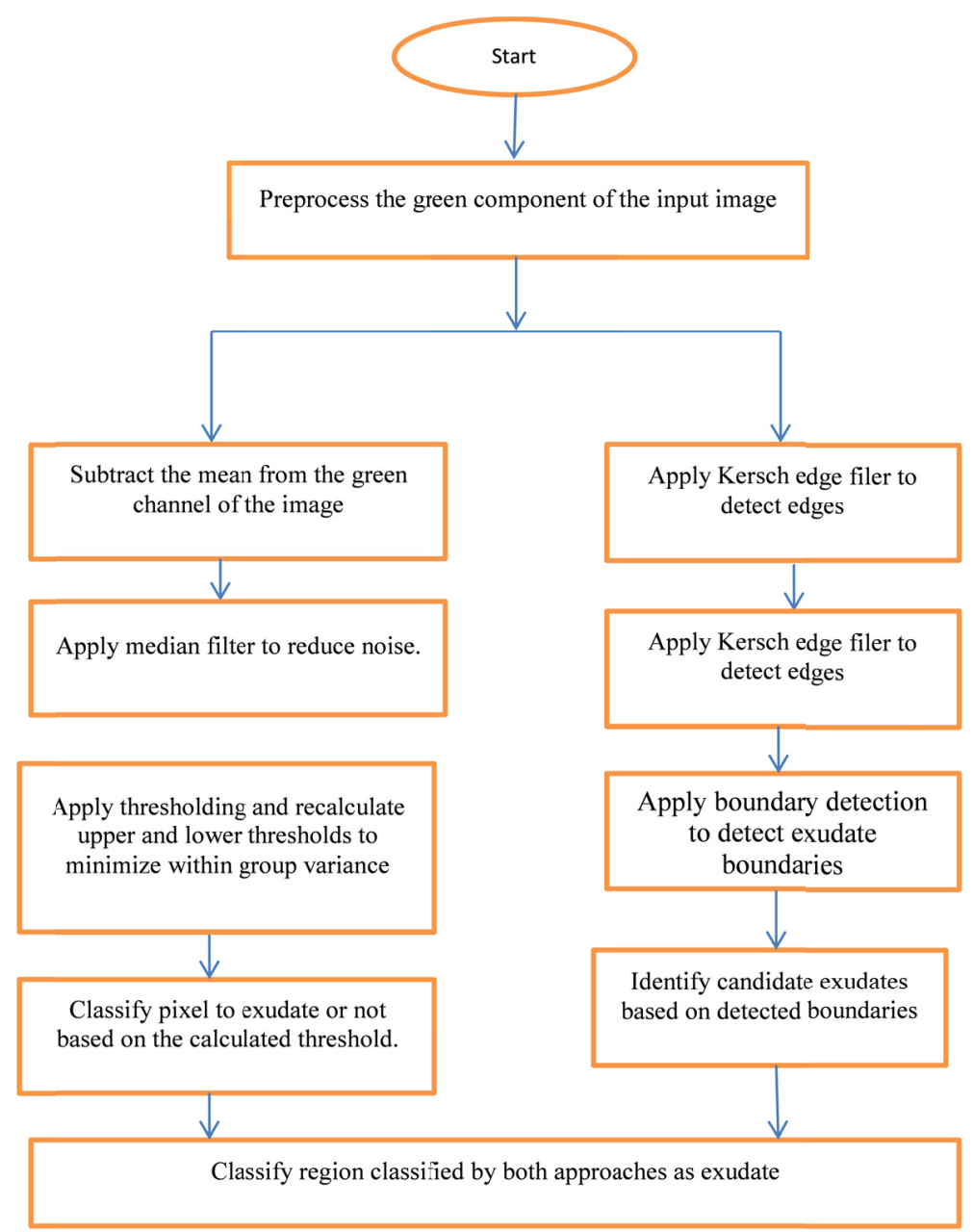

Figure 6 . The proposed al gorithm to detect exudates in eye fundus image

\section{Implementation and Experimental Results}

The proposed algorithm for exudates detection is implemented and tested using a known set of eye fundus retina images DIARETDB0 (Kauppi et al., 2006). DIARETDB0 is database for benchmarking contains 130 color images of size 1500 X 1152 pixels. Each image in this database has ground truth information about the exudates in the retinal images.

We implemented the proposed algorithm using MATLAB. Figure 7 shows a set of retinal images and the corresponding image with marked exudates. Table 1 summarizes the results obtained by the proposed algorithm for sample images selected from the database. This table provides us with the number of exudates found in the image, and the percent of the area of exudates to the area of eye fundus retina.

In order to evaluate our algorithm, we asked an expert in this field to identify the exudates manually. The performance of the proposed algorithm is evaluated using the sensitivity and specificity which defined as follows:

$$
\text { sensitivity }=\frac{T_{P}}{T_{P}+F_{N}}
$$

and

$$
\text { specificity }_{I}=\frac{T_{N}}{T_{N}+F_{P}}
$$



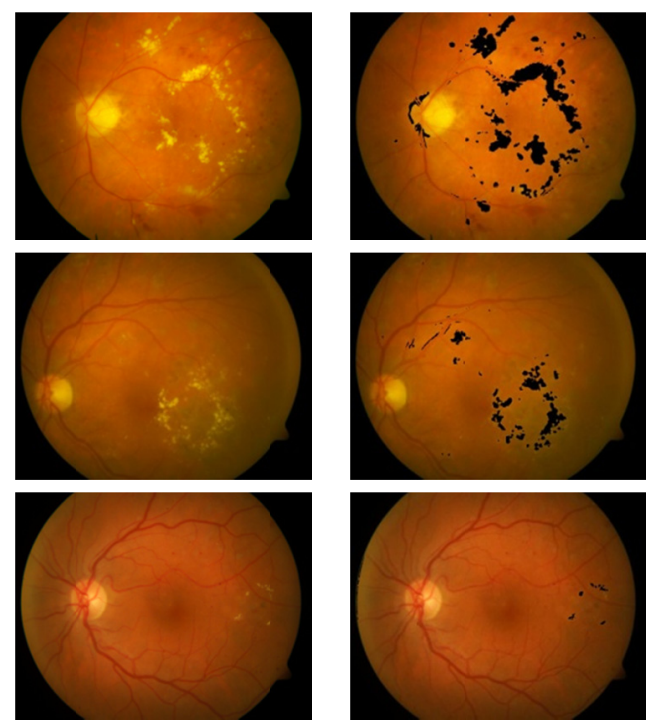

Figure 7. Sample image from DIARETDB0 database and their corresponding image with marked exudates as detected by the proposed algorithm

Where $T_{P}$ is the area (in pixels) that truly identified exudates, $F_{N}$ is the area of exudates marked by the algorithm as normal, $F_{P}$ is an area marked falsely as exudates, and $T_{N}$ is the area truly identified as normal. The calculated sensitivity for all images in DIARETDB0 database is calculated. A sample of the results for the sensitivity is shown in Table 1. The average sensitivity for all images is $92.1 \%$ and the average specificity $_{I}$ is $99.1 \%$. Table 2 summarizes the sensitivity for our algorithm and other published algorithm in the literature. This table concluded that the proposed algorithm performance is the best.

Table 1. Sample result obtained by our algorithm for randomly selected images

\begin{tabular}{cccc}
\hline Image number & $\#$ of exudates & Exudate area percent & sensitivity $_{I}$ \\
\hline 1 & 115 & $6.970 \%$ & $97.3 \%$ \\
2 & 74 & $2.416 \%$ & $93.2 \%$ \\
3 & 38 & $0.345 \%$ & $92.1 \%$ \\
4 & 20 & $0.229 \%$ & $90.5 \%$ \\
5 & 164 & $1.696 \%$ & $89.7 \%$ \\
6 & 21 & $0.146 \%$ & $88.9 \%$ \\
7 & 54 & $0.258 \%$ & $89.3 \%$ \\
8 & 27 & $0.179 \%$ & $90.2 \%$ \\
9 & 16 & $0.021 \%$ & $90.3 \%$ \\
10 & 11 & $0.021 \%$ & $91.6 \%$ \\
\hline
\end{tabular}

The calculation of sensitivity and specificity based on pixel classification is impartment for comparison between different algorithms. For physicians, it is better to classify images as normal or abnormal. Abnormal image is an image that contains exudates. Abnormal cases may need medication and follow up. Therefore, we redefined sensitivity and specificity according to (Kauppi et al., 2006) as follows:

$$
\text { sensitivity }_{D}=\frac{T_{P a}}{T_{P a}+F_{N a}}
$$

and,

$$
\text { specificity }_{\mathrm{D}}=\frac{T_{N a}}{T_{N a}+F_{P a}}
$$

Where $T_{P a}$ is the number of abnormal fundus images found as abnormal, $T_{N a}$ is the number of normal fundus 
images found as normal, $F_{P a}$ is the number of normal fundus images found as abnormal, and $F_{N a}$ is the number of abnormal fundus images found as normal (Kauppi et al., 2006). The sensitivity $_{D}$ and specificity $_{D}$ is computed for our proposed algorithm using DIARETDB0. The specificity is $89.2 \%$ and the sensitivity is $92.3 \%$. Kauppi et al evaluated sensitivity ${ }_{D}$ and specificity $_{D}$ for algorithm developed by Kuivalainen (2005). The sensitivity $y_{D}$ and specificity $_{D}$ was $79 \%$, and $58 \%$ respectively. It is very clear that our algorithm performance is better.

Table 2. Comparison between our algorithm and recent published approaches utilizing sensitivity measure

\begin{tabular}{ll}
\hline Algorithm & sensitivity $_{I}$ \\
\hline Our algorithm & $92.1 \%$ \\
Walter et al. & $66.0 \%$ \\
Welfer et al. & $70.48 \%$ \\
S'anchez et al. & $88 \%$ \\
\hline
\end{tabular}

\section{Conclusions}

Eye fundus image analysis for diabetic disease plays a major role for evaluating the development diabetic retinopathy. This paper introduces a new approach to detect exudates, which is the main component to measure the development of diabetic retinopathy. In our approach, we applied two different techniques to identify exudates. The first one relies on color feature of exudate, and the second approach utilizes edge and boundaries to identify exudates. The proposed algorithm is implemented and tested using a known eye fundus database images (DIARETDB0). The performance of the algorithm is evaluated using sensitivity and specificity. Our algorithm average sensitivity is equal to $92.1 \%$. The average specificity is more than $99 \%$. The results of evaluation showed that the proposed algorithm achieved better results in comparison with recently published algorithms.

\section{References}

Agurto, C., Murray, V., Barriga, E., Murillo, S., Pattichis, M., Davis, H., ... Soliz, P. (2010). Multiscale AM-FM methods for diabetic retinopathy lesion detection. IEEE Transaction on Medical Imaging, 29, 502-512. http://dx.doi.org/10.1109/TMI.2009.2037146

Arbel'aez, P., Maire, M., Fowlkes, C., \& Malik, J. (2011). Contour detection and hierarchical image segmentation. Pattern Analysis and Machine Intelligence, 33(5), 898-916. http://dx.doi.org/10.1109/TPAMI.2010.161

Cunha-Vaz, J. (1998). Diabetic macular edema. European Journal of Ophthalmol, 8(3), 127-130.

Garcia, M., Sanchez, C., Lopez, M., Abasolo, D., \& Hornero, R. (2009). Neural network based detection of hard exudates in retinal images. Computer Methods and Programs in Biomedicine, 93, 9-19. http://dx.doi.org/10.1016/j.cmpb.2008.07.006

Geetan, T., Acharya, R., \& Ng, E. (2008). Automated identification of eye diseases using higher-order spectra. Journal of Mechanics in Medicine and Biology, 8(1), 121-136. http://dx.doi.org/10.1142/S0219519408002504

Kauppi, T., Kalesnykiene, V., Kamarainen, J. K., Lensu, L., Sorri, I., Uusitalo, H., ... Pietilä, J. (2006). DIARETDB0: Evaluation Database and Methodology for Diabetic Retinopathy Algorithms. Technical report.

Kuivalainen, M. (2005). Retinal image analysis using machine vision. Master's thesis, Lappeenranta University of technology, Finland.

Liang, Y., \& Yin, Y. (2013). A new multilevel thresholding approach based on the ant colony system and the EM algorithm. International Journal of Innovative Computing, Information, and Control, 9(1), 319-337.

Niemeijer, M., van Ginneken, B., Russell, S., \& Abr'amoff, M. (2007). Automated detection and differentiation of drusen, exudates, and cotton-wool spots in digital color fundus photographs for diabetic retinopathy diagnosis. Investigative Ophthalmology and Visual Science, 48(5), 2260-2267. http://dx.doi.org/10.1167/iovs.06-0996

Sanchez, C., Garcia, M., Mayo, A., Lopez, M., \& Hornero, R. (2009). Retinal image analysis based on mixture models to detect hard exudates. Medical Image Analysis, 13, 650-658. http://dx.doi.org/10.1016/j.media.2009.05.005 
Schneider, C., Rasband, W., \& Eliceiri, K. (2012). NIH Image to ImageJ: 25 years of image analysis. Nature methods, 9(7), 670-675. http://dx.doi.org/10.1038/nmeth.2089

Tasman, W., \& Jaeger, E. (2001). The Wills Eye Hospital: Atlas of Clinical Ophthalmology (2nd ed.). Lippincott Williams and Wilkins Publisher.

Walter, T., Klein, J., Massin, P., \& Erginay, A. (2002). A Contribution of Image Processing to the Diagnosis of Diabetic Retinopathy - Detection of Exudates in Color Fundus Images of the Human Retina. IEEE Trans. On Medical Imaging, 21(10), 1236-1243. http://dx.doi.org/10.1109/TMI.2002.806290

Welfera, D., Scharcanskia, J., \& Marinho, D. (2010). A coarse-to-fine strategy for automatically detecting exudates in color eye fundus images. Computerized Medical Imaging and Graphics, 34, 228-235. http://dx.doi.org/10.1016/j.compmedimag.2009.10.001

Xu, L., \& Luo, S. (2009). Support vector machine based method for identifying hard exudates in retinal images. Proceedings of the IEEE Youth Conference on Information, Computing and Telecommunication (YC-ICT2009) (pp. 138-141).

\section{Copyrights}

Copyright for this article is retained by the author(s), with first publication rights granted to the journal.

This is an open-access article distributed under the terms and conditions of the Creative Commons Attribution license (http://creativecommons.org/licenses/by/3.0/). 